\title{
The GIS Management Tool Was Used in the Tirana Earthquake on November 26, 2019
}

\author{
Genc Salja ${ }^{1}$, Marpol Koço ${ }^{1}$ \\ ${ }^{1}$ Department of Geodesy, Faculty of Civil Engineering, Polytechnic University of Tirana, Rr. Muhamet Gjollesha, Tirana, Albania \\ salja.genc@gmail.com \\ ${ }^{1}$ Department of Applied Geology, Environment and Geoinformatics, Faculty of Geology and Mine, Polytechnic University of Tirana, \\ Rr.Elbasanit, Tirana, Albania \\ marjo_peko87@hotmail.com
}

\begin{abstract}
On the 26th of November 2019, an earthquake of moment magnitude 6.4 struck the northwest region of Albania. This paper summarizes the development of a Geographic Information System "GIS" for the management and analysis of information on damaged facilities in Tirana during survey. The application of GIS techniques allowed us to store, extract, modify and analyze data on earthquake-damaged buildings. The final product consists of information converted as maps, as well as numerical tables associated with the maps. The study was conducted using QGIS and ArcGIS software and was structured in thematic vector and Raster. The damaged sites are placed on a map, with all the information on the earthquake (number of dead, number of houses collapsed, economic losses.) Accompanied by relevant data. GIS is structured in such a way as to be able to integrate with other geospatial data from other local and central institutions. Furthermore, the GIS system enables various spatial and statistical analyzes, can provide support both for damage assessment, its geographical distribution determination, identification of regions repeatedly affected by seismic events, quantify the level of seismic and extends the assessment to other natural hazards. These applications were a GIS portal site for quickly sharing disaster-related GIS data, an efficient method of accessing and recording damage in the field throughout the city.
\end{abstract}

Keywords: Albania earthquake $\cdot$ GIS, Field survey $\cdot$ Post-earthquake $\cdot$ Building's damage assessment

\section{Introduction}

On Saturday 21st September 2019 at 15:15 CET, an earthquake of moment magnitude (Mw) 5.6 and shallow focal depth $(\sim 10 \mathrm{~km})$ (Bilgin and Hysenlliu 2020; USGS 2020) hit the northwest region of Albania. The epicenter of the earthquake was estimated $5 \mathrm{~km}$ northwest Durrës city ( $35 \mathrm{~km}$ west of Tirana). Despite the proximity to the city, the event had relatively small consequences with no fatalities, i.e., 110 injured people and 120 damaged buildings without structural failures. This was the first event of the seismic sequence and, on the 26th of November, at 03:54 CET, central and north-west Albania was struck by the main shock of the sequence with Mw 6.4 and shallow focal depth ( $20 \mathrm{~km}$ ) (USGS 2020). The epicenter was $16 \mathrm{~km}$ westsouthwest of the town of Mamurras in Kurbin municipality. The earthquake was felt strongly in Albania's capital Tirana where the significant duration (bracketed between the 5\% and $95 \%$ of Arias intensity) of the strong motion was $24 \mathrm{~s}$. The earthquake was also felt in places as far away as Taranto (Italy) and Belgrade (Serbia). A total of 51 people were killed in the earthquake, with about 3,000 injured. The first estimates revealed that the total effect of the disaster in the 11 municipalities amounts to 985 million euro, including direct and indirect losses (Republic of Albania Council of Ministers 2020). In Figure 1 are shown the epicenters of earthquakes sequences and the location of Tirana Municipality.

We had several damaged buildings to inspect, and many field surveyor's groups. This brought a lot of information in a very short time.

We chose to develop a GIS Application to the disaster response on the damage caused by the Earthquake in the Municipality of Tirana, which, in terms of quantity and quality of known data, can be used as an effective tool for creating an integrated information system which allows a comparison of data from different sources of information in a quick time. An indirect analysis of these data allows us to access the damage, to determine its geographical distribution, to identify areas repeatedly affected by seismic events, and to quantify the level of seismic damage in the area.

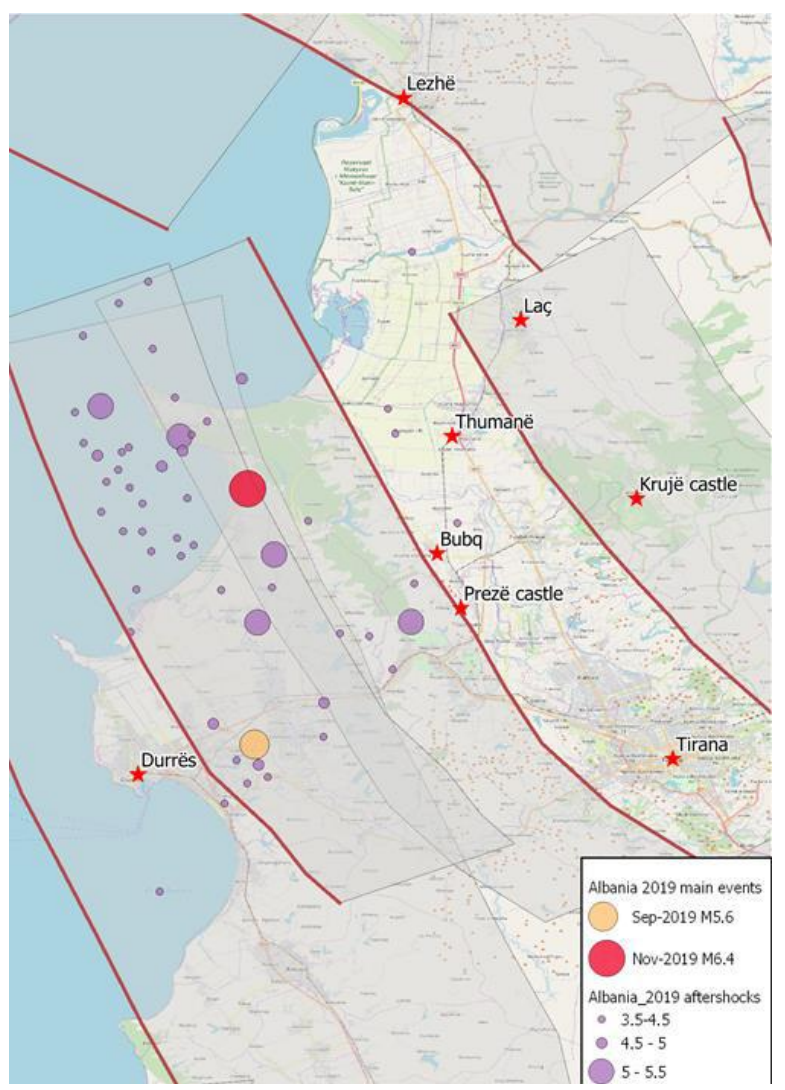

Figure 1: Durres earthquakes sequence and distance from Tirana (Adapted from EEFIT). 


\section{Data Formatting in GIS}

Since we need to store the data for each Building as a Basic Layer, we used the geo-data from address system. During the on-site inspections it was noticed that the data information was not accurate two or more buildings were digitized as one and in some cases the buildings were missing. To add the missing buildings, some additional data were used such as Orthophoto of 2018, Orthophoto of 2015, Satellite Imaging, etc. A Database was created which would store the Field Reported data, as shown in Table 1.

Table 1: Preliminary Database

\begin{tabular}{|l|}
\hline Inspection Group \\
\hline Date of Inspection \\
\hline Object Address \\
\hline Object Contact Person \\
\hline Telephone Number of the Contact Person in the Facility \\
\hline Coordinates N - (Latitude) \\
\hline Coordinates E - (Longitude) \\
\hline Object Typology \\
\hline Structure Typology \\
\hline The height of the building on the floors \\
\hline Field Typology \\
\hline Year of Construction \\
\hline Structural Damage \\
\hline Details on structural damages as well as other damages \\
\hline Advice on Further Actions to Take to Secure the Facility \\
\hline Forced evacuation of the Facility \\
\hline Typology of Foundations / Basement \\
\hline
\end{tabular}

For each of the Fields there were categorizes and subcategories. In the first week of the damage assessment survey there was no Standard format from the Government for determining damage assessment. Caused by the Earthquake. We had problem determine the location of the building as there were no addresses sign in the building in some cases even in the street sign in the field, so to determine the position of the building the coordinates of the building were find via Mobile Phones in the WGS84 Geographic System. These data were regularly discarded by the operators then in the Municipality of Tirana. The operators in the Municipality, reading the damage assessment, made categorizations by interpreting the Findings Act in 3 Categories: Safe; Review; Evacuation.

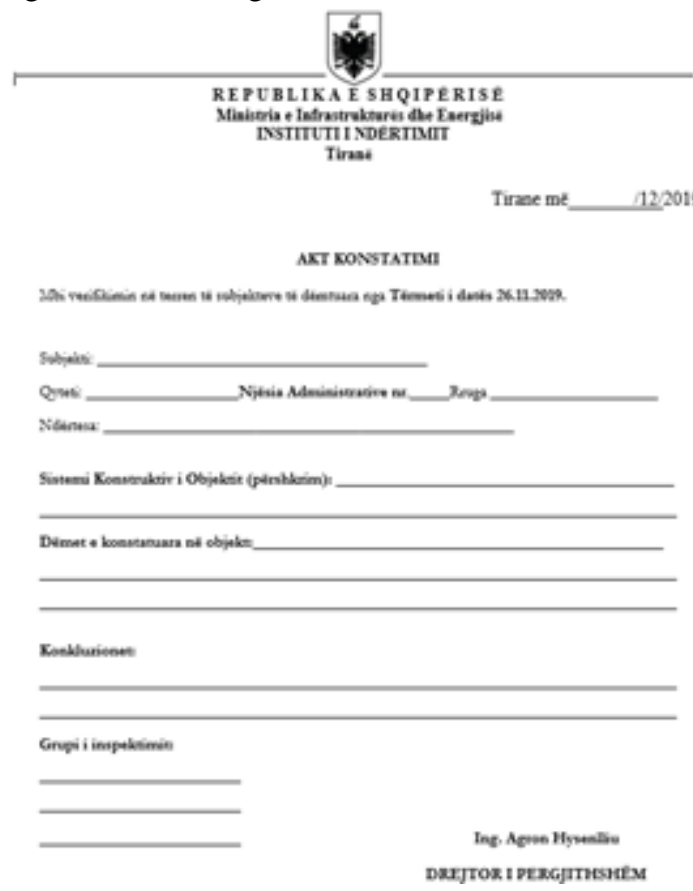

Figure 2: The first model of the damage assessment

It was soon noticed by the Government that through this format it was very difficult to determine the damages and to build a database which would show us the buildings which needed to be evacuated immediately or which required immediate intervention.

On 8 December 2019, The Inter-ministerial COMMISSION ON CIVIL EMERGENCY after several discussions on collaboration with UNDP and other agencies drafted the new Standard for damage assessment shown in Figure 3.

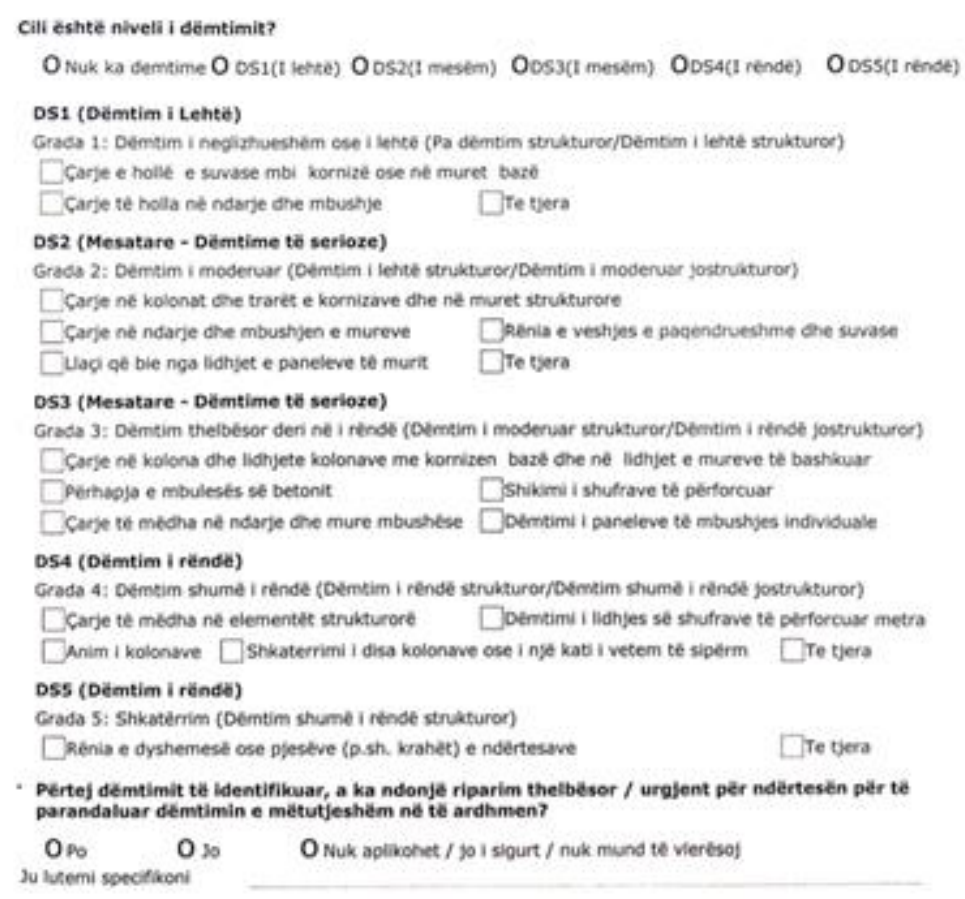

Figure 3: The second model of the damage assessment 
Through this model was made Standard damage assessment which considered the damage states:

- DS0 (No Damage)

- DS1 (Light Damage)

- DS2 (Medium-Serios Damage)

- DS3 (Medium-Serios Damage)

- DS4 (Heavy Damage)

- DS5 (Heavy Dagame)

\section{GIS Project and Structure}

GIS is used as data storage. GIS includes maps for the entire Municipality of Tirana. Spatial database built into the
KRRGJSH system EPSG: 6870 is the basis for all data to be in a single georeferenced coordinate system. Earthquake damages were stored as points with fixed coordinates, each point above is placed with a building which shows damage level also features of the building.

Orthophoto is a useful reference to show the actual condition of the territory. The data captured from the damage assessment on the field were completed in an excel spreadsheet and then dumped into GIS. Each data had Coordinates N, E. This methodology is shown in the Diagram below.

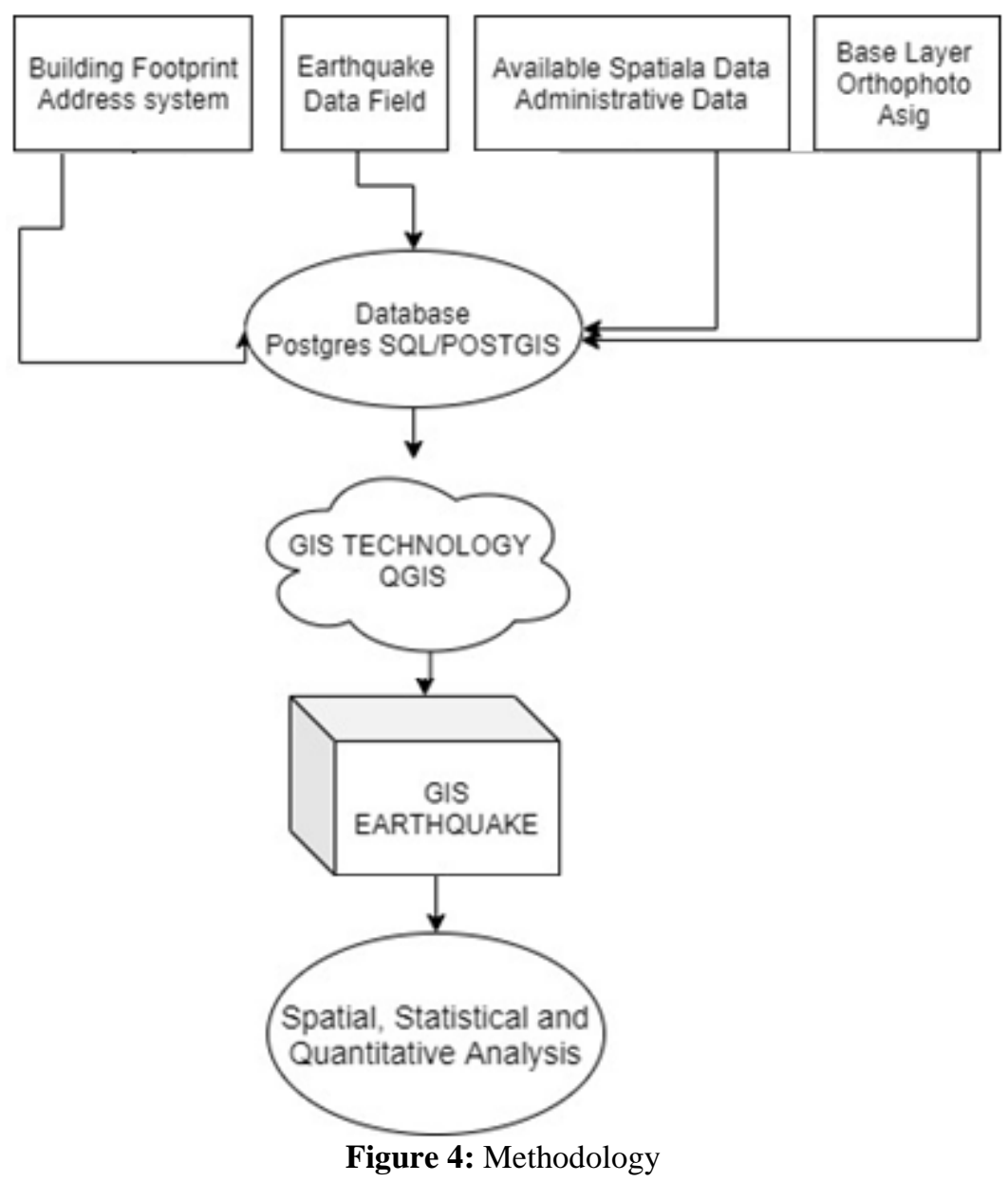

Through this spatial database created we can store any data on buildings damaged by the earthquake with location as well as geographical reference with other elements such as Cadaster, Building Permits, Geology, etc.

Through the software QGIS we can do the management of this data, the presentation of this data and their statistical analysis based on different geographical divisions. helps us to build interpretive summary, can provide support for damage assessment, its geographical determination, distribution, identification of regions continuously affected by seismic events.

Earthquake maps and tables will be used for government intervention in the areas most affected by the earthquake as well as policies for Allocation of Funds needed for their Reconstruction.

\section{Data Consultation and Submission}

The main advantages of GIS software consist in its ease use and instant access to real-time information. Damaged places were automatically digitized on the map by entering the coordinates of points $\mathrm{N}$, E, each point constitutes and represents a damaged object. Using a UNIQUE identification code which was later used for reference as the GIS CODE Object Address, the damage data was linked to individual points, displaying a one-to-one correspondence on the map.

Linking the data to the map allows thematic maps to be generated within the initial GIS processing. In the specific case, linking the dots to the damage data. The structure and operation of GIS allows many automated operations to be performed for the integrated representation of different types of data, such as the overlap of several thematic maps, 
namely geological and tectonic maps Elevation Maps DEM and Orthophoto, which can show the contribution of geology and tectonic structures up to the intensity of the earthquake.
Distributions of earthquake damage by Administrative Units is shown in Figure 5.

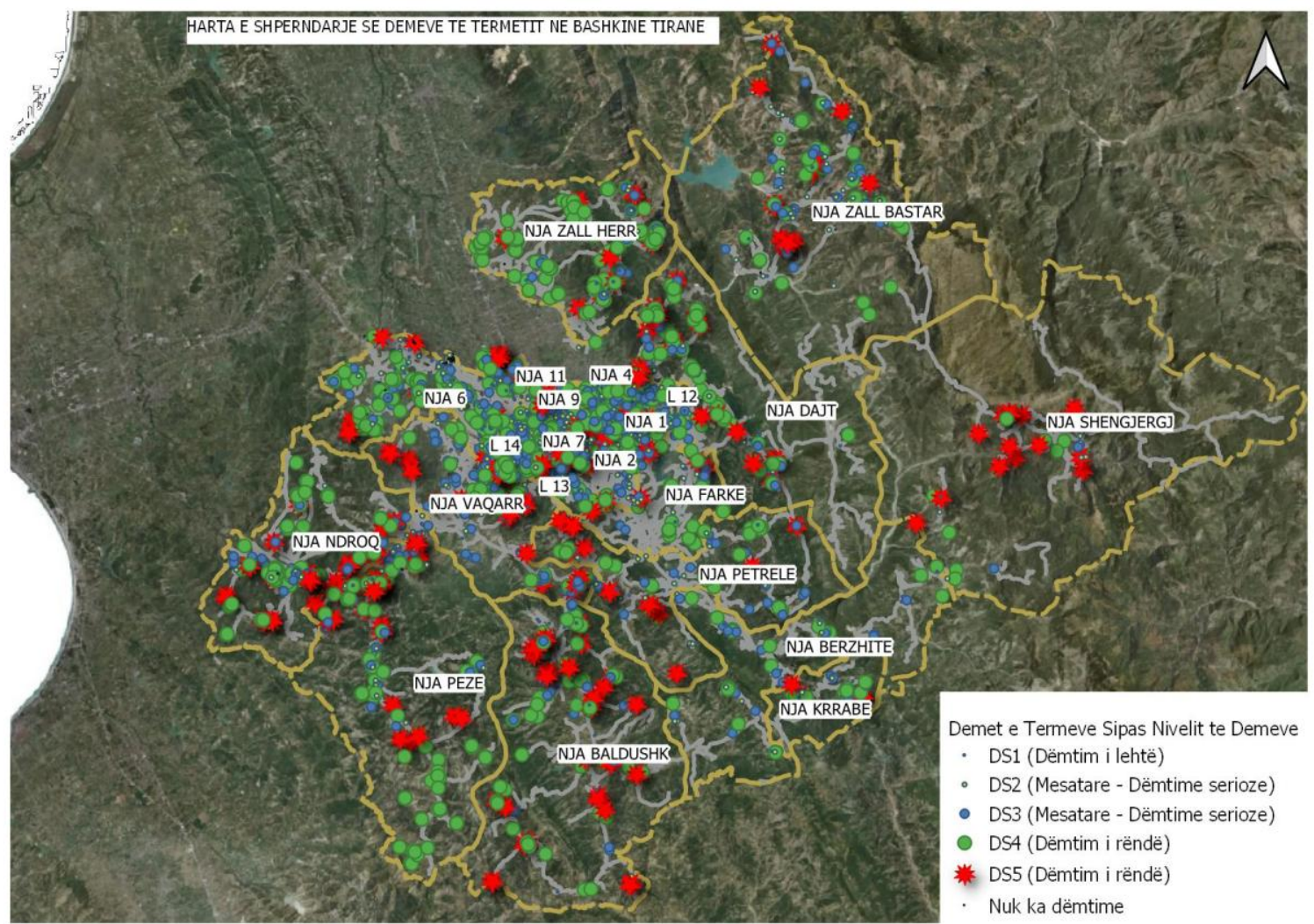

Figure 5: Distribution of damages in Administrative Units of Tirana

Another interesting map is the Heat map according to the geographical distribution shows the accumulative distribution of damages as a weight for the generation is used the parameter if the building is habitable or NO. It can be noticed from Figure 6 that the Administrative Unit (NJ) 6 and 10 have experienced the biggest damages.

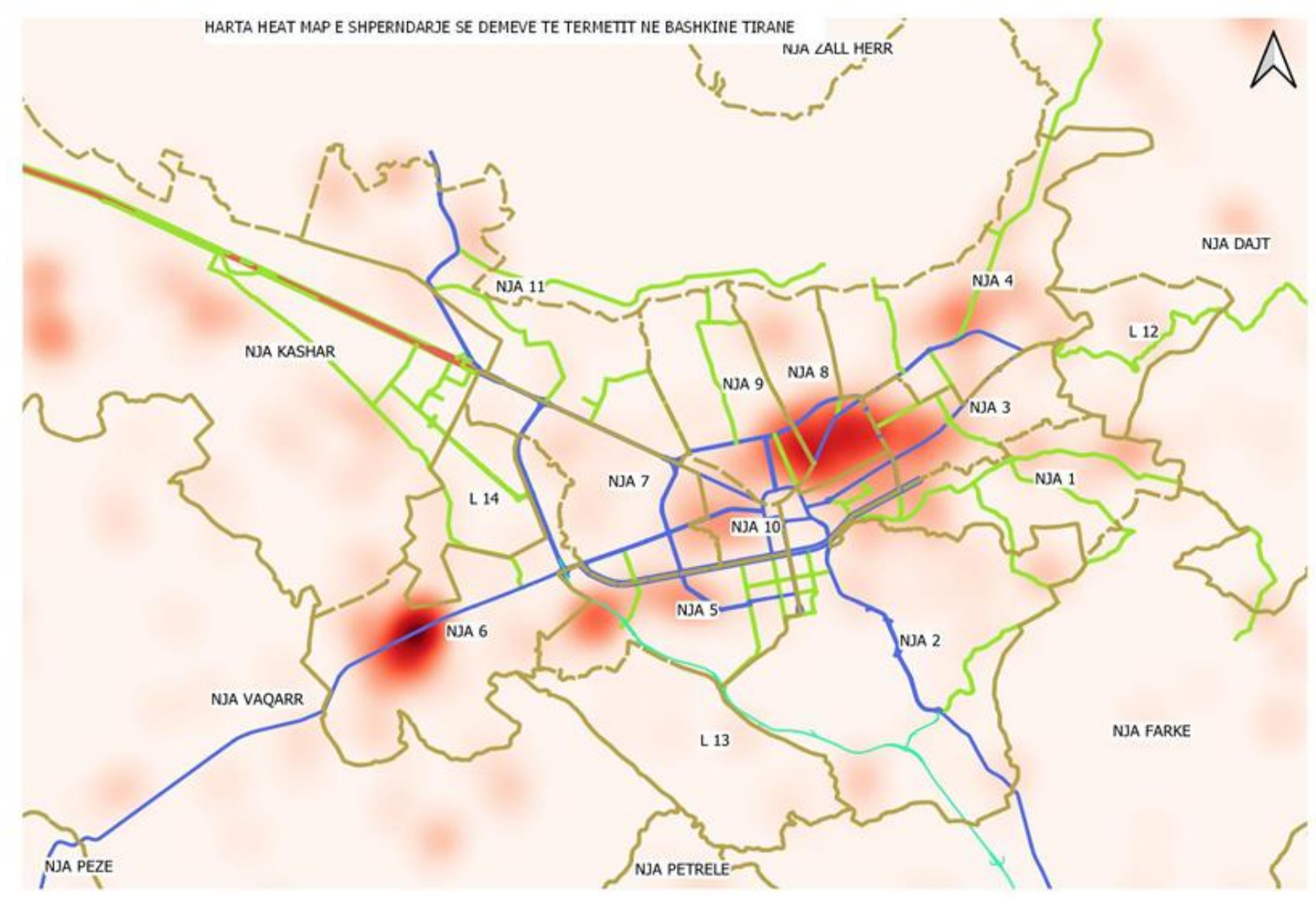

Figure 6: HeatMap of Administrative Units of Tirana Municipality 
Another interesting map is the map distribution of damage within the territory of Tirana municipality as shown in
Figure 7. We see that the terrain has not had any clear effect on the damages of the buildings.

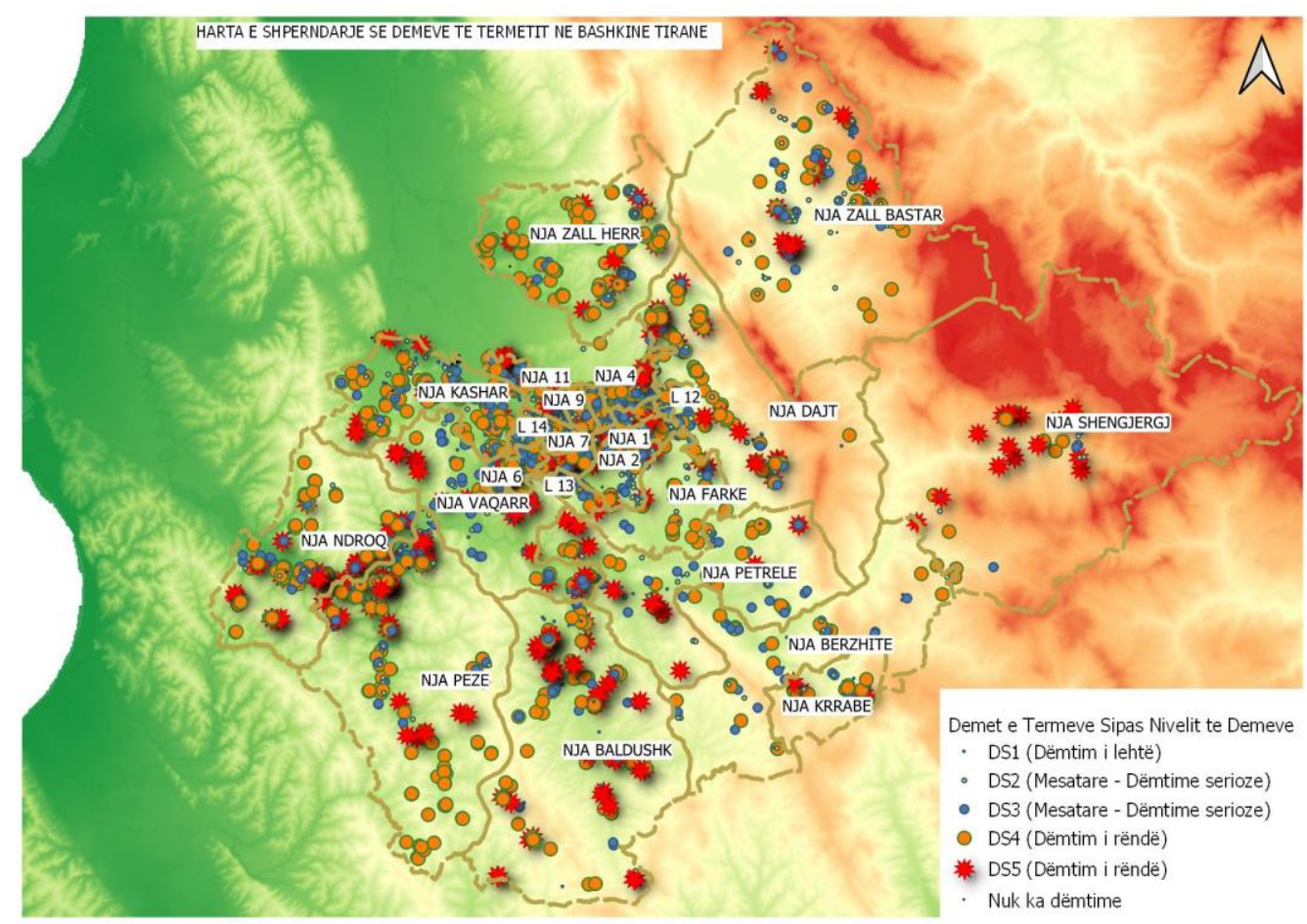

Figure 7: Damage map as per different DS based on terrain digital model.

\section{Results and Discussions}

From the verifications the biggest damages are concentrated in the Administrative Unit6, Administrative Unit 8, Administrative Unit 9, Administrative unit 5 and Administrative Unit 3 which also show a large concentration of damages. To make an analysis of the castings it is necessary for the Structural Engineers to look at the data of the buildings such as the types of damages, the materials, the geological map. From the results in the Database, it appears that over 5185 Inspections of Buildings in Tirana with Damage have been performed. From the Statistics it appears that the approximate data are summarized in Table 2.

Table 2: Statistical data of the damaged buildings

\begin{tabular}{|c|c|c|c|}
\hline & \multicolumn{3}{|c|}{ Statistic Data } \\
\hline & FID $=$ & niveli_dem & FREQUENCY \\
\hline 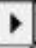 & 1 & Te Pa inspektuara & 78542 \\
\hline & 2 & DS1 (Dëmtim i lehtê) & 1295 \\
\hline & 3 & DS2 (Mesatare - Dëmtime serioze) & 1197 \\
\hline & 4 & DS3 (Mesatare - Dèmtime serioze) & 971 \\
\hline & 5 & DS4 (Dëmtim i rēndẻ) & 1145 \\
\hline & 6 & DS5 (Dëmtim i rẽndè) & 277 \\
\hline & 7 & Nuk ka dëmtime & 300 \\
\hline
\end{tabular}

It should be quoted that these have been the first data from the Field. Based on these data then for buildings with damage DS4-DS5 In-depth structural assessment was conducted by the Institute of Construction or Private Structural Design Companies.

\section{Conclusions}

GIS is a useful tool in natural disaster management.

It serves for the clear identification of damages in a more accurate and efficient way, it serves for the advance planning of the Objects for expertise, for informing the multilateral actors for the evacuation of the inhabitants in an immediate manner in case of need, for the planning of the budget in advance precisely. By making the combinations of Earthquake and Civil Status data, ACA has also made it possible to create preliminary reports on the funds needed for the Rebuilding.

In the process of Reconstruction GIS has been the tool used to calculate the bill of damages for EU funding, with a tool Transparent which can be accessed from any country at the Micro or Macro level.

\section{References}

[1] https://rindertimi.gov.al/

[2] http://www.instat.gov.al/

[3] https://www.esri.com/content/dam/esrisites/sitecorearchive/Files/Pdfs/library/bestpractices/earthquakes.pd $\mathrm{f}$

[4] https://www.undp.org/

[5] https://gis.tirana.al/

[6] https://www.esri.com/en-us/home

[7] https://qgis.org/en/site/

[8] http://asig.gov.al/

[9] EARTHQUAKE DISASTER GIS AND SPATIALTEMPORAL PATTERN OF EARTHQUAKE 
DISASTERS IN CHINA1 Mao Feng and Ma Jin feng and Zhou Wen sheng

[10] THE MW6.4 ALBANIA EARTHQUAKE ON THE 26TH of NOVEMBER 2019 A FIELD REPORT BY EEFIT OCTOBER 2020

[11] Anton Andonov, Stoyan Andreev, Fabio Freddi, Enes Veliu,

[12] Federica Greco, Roberto Gentile, Viviana Novelli, Cardiff University

[13] Observations from the 26th of November 2019 Albania earthquake: the earthquake engineering field investigation team (EEFIT) mission.

[14] Fabio Freddi1 · Viviana Novelli2 - Roberto Gentile1,3 - Enes Veliu4 · Stoyan Andreev5 .

[15] Anton Andonov5 · Federica Greco6 · Emiljano Zhuleku7

\section{Author Profile}

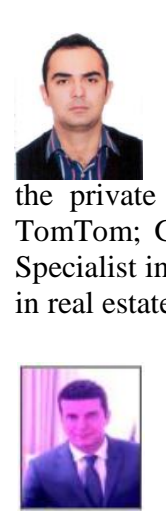

Phd.Cand. Eng. Genc SALJA: Born in Shkodra, 1989.Lives in Tirana. Completed studies as Geodetic Engineering on 2012. He started PhD studies on 2014.

His work experience includes: Geodetic engineer in the private sector, GIS Expertise for EU Project, Freelancerfor TomTom; Guest Lecturer at the Polytechnic Universityof Tirana; Specialist in the GIS/CadasterSector in Tirana Municipality. Expert in real estate valuation.

Phd.Cand. Eng. Marpol KOÇO, Born in Tirana, 1987. Finished study as Mining Survey Engineer on 2011. He started PhD studies on 2012. His work experience includes Geodetic engineer in private sector; External Lecture at University; Specialist in the Directorate of Policies and Mining Development; Head of Sector in Cadastral Directorate in Tirana Municipality. Expert real estate valuation. 